\title{
Nucleon electromagnetic form factors and electroexcitation of low lying nucleon resonances in a light-front relativistic quark model
}

\author{
I.G. Aznauryan ${ }^{1,2}$ and V.D. Burkert ${ }^{1}$ \\ 1 Thomas Jefferson National Accelerator Facility, Newport News, Virginia 23606, USA \\ ${ }^{2}$ Yerevan Physics Institute, 375036 Yerevan, Armenia
}

\begin{abstract}
We utilize a light-front relativistic quark model (LF RQM) to predict the $3 q$ core contribution to the electroexcitation amplitudes for the $\Delta(1232) \mathrm{P}_{33}, \mathrm{~N}(1440) \mathrm{P}_{11}, \mathrm{~N}(1520) \mathrm{D}_{13}$, and $\mathrm{N}(1535) \mathrm{S}_{11}$ up to $Q^{2}=12 \mathrm{GeV}^{2}$. The parameters of the model have been specified via description of the nucleon electromagnetic form factors in the approach that combines $3 q$ and pion-cloud contributions in the LF dynamics.

PACS numbers: 12.39.Ki, 13.40.Gp, 13.40.Hq, 14.20.Gk
\end{abstract}

\section{INTRODUCTION}

In the past decade, with the advent of the new generation of electron beam facilities, there has been dramatic progress in studies of the electroexcitation of nucleon resonances that resulted in more reliable extractions of the resonance electrocouplings, and a significant extension of the $Q^{2}$ range. The most accurate and complete information has been obtained for the four lowest excited states, which have been measured in a range of $Q^{2}$ up to $8 \mathrm{GeV}^{2}$ for the $\Delta(1232) P_{33}$ and $N(1535) S_{11}$, and up to $4.5 \mathrm{GeV}^{2}$ for the $N(1440) P_{11}$ and $N(1520) D_{13}$ (see reviews [1, 2]).

At relatively small $Q^{2}$, nearly massless pions generate pion-loop contributions that may significantly alter quark model predictions. It is expected that the corresponding hadronic component, including contributions from other mesons, will be rapidly losing strength with increasing $Q^{2}$. The Jefferson Lab $12 \mathrm{GeV}$ upgrade will open up a new era in the exploration of excited nucleons when the quark core of the nucleon and its excited states will be more fully exposed to the electromagnetic probe.

The aim of this paper is to estimate the $3 q$ core contribution to the electrocoupling amplitudes of the $\Delta(1232) P_{33}, N(1440) P_{11}, N(1520) D_{13}$, and $N(1535) S_{11}$. The approach we use is based on the LF dynamics which presents the most suitable framework for describing the transitions between relativistic bound systems $[3-5]$. In early works by Berestetsky and Terent'ev [4], the approach was based on the construction of the generators of the Poincaré group in the LF. It was later formulated in the infinite momentum frame (IMF) [6, 7]. This allowed one to demonstrate more clearly that diagrams which violate impulse approximation, i.e. the diagrams containing vertices like $\gamma^{*} \rightarrow q \bar{q}$, do not contribute. The interpretation of results for $\gamma^{*} N \rightarrow N\left(N^{*}\right)$ in terms of the vertices $N\left(N^{*}\right) \leftrightarrow 3 q$ and corresponding wave functions became more evident. In Refs. [7-10], the LF RQM formulated in IMF was utilized for the investigation of nucleon form factors and the electroexcitation of nucleon resonances. These observables were investigated also in the LF Hamiltonian dynamics in Ref. [11]. In both cases a complete orthogonal set of wave functions has been used, that correspond to the classification of the nucleon and nucleon resonances within the group $S U(6) \times O(3)$; the relativistic-covariant form of these wave functions has been found in Ref. [7]. We specify the parameters of the model for the $3 q$ contribution via description of the electromagnetic form factors, by combining the $3 q$ and pion-cloud contributions. The pion-cloud contribution has been incorporated using the LF approach of Ref. [12].

In Sec. II we present briefly the formalism to compute the $3 q$ contribution to the $\gamma^{*} N \rightarrow N\left(N^{*}\right)$ amplitudes. In Sec. III we discuss the description of nucleon electromagnetic form factors at $0 \leq Q^{2}<16 \mathrm{GeV}^{2}$. To achieve description of experimental data at $Q^{2}>0$, we incorporate the $Q^{2}$-dependence of the constituent quark mass that is expected from the lattice QCD and Dyson-Schwinger equations approach [13 15]. With the LF RQM specified via description of the nucleon electromagnetic form factors, we predict in Sec. IV the quark core contribution to the electroexcitation amplitudes of the aforementioned resonances at $Q^{2} \leq 12 \mathrm{GeV}^{2}$. The results are summarized in Sec. V.

\section{QUARK CORE CONTRIBUTION TO TRANSITION AMPLITUDES}

The $3 q$ contribution to the $\gamma^{*} N \rightarrow N\left(N^{*}\right)$ transitions has been evaluated within the approach of Refs. 7, 8] where the LF RQM is formulated in the IMF. The IMF is chosen in such a way, that the initial hadron moves along the $z$-axis with the momentum $P_{z} \rightarrow \infty$, the virtual photon momentum is $k^{\mu}=$ $\left(\frac{m_{\text {out }}^{2}-m_{i n}^{2}-\mathbf{Q}_{\perp}^{2}}{4 P_{z}}, \mathbf{Q}_{\perp},-\frac{m_{\text {out }}^{2}-m_{i n}^{2}-\mathbf{Q}_{\perp}^{2}}{4 P_{z}}\right)$, the final hadron momentum is $P^{\prime}=P+k$, and $Q^{2} \equiv-k^{2}=\mathbf{Q}_{\perp}^{2} ; m_{i n}$ and $m_{\text {out }}$ are masses of the initial and final hadrons, respectively. The matrix elements of the electromagnetic current are related to the $3 q$-wave functions in the following way:

$$
\begin{aligned}
& \frac{1}{2 P_{z}}<N\left(N^{*}\right), S_{z}^{\prime}\left|J_{e m}^{0,3}\right| N, S_{z}>\left.\right|_{P_{z} \rightarrow \infty} \\
& =3 e \int \Psi^{\prime+}\left(p_{a}^{\prime}, p_{b}^{\prime}, p_{c}^{\prime}\right) Q_{a} \Psi\left(p_{a}, p_{b}, p_{c}\right) d \Gamma,
\end{aligned}
$$


where $S_{z}$ and $S_{z}^{\prime}$ are the projections of the hadron spins on the $z$-direction. In Eq. (11), it is supposed that the photon interacts with quark $a$ (the quarks in hadrons are denoted by $a, b, c), Q_{a}$ is the charge of this quark in units of $e\left(e^{2} / 4 \pi=1 / 137\right) ; \Psi$ and $\Psi^{\prime}$ are wave functions in the vertices $N\left(N^{*}\right) \leftrightarrow 3 q ; p_{i}$ and $p_{i}^{\prime}(i=a, b, c)$ are the quark momenta; $d \Gamma$ is the phase space volume. The relations between the matrix elements (11) and the $\gamma^{*} N \rightarrow N\left(N^{*}\right)$ form factors and transition helicity amplitudes are given in Appendix.

We parametrize the quark momenta in the IMF via:

$$
\begin{aligned}
& \mathbf{p}_{i}=x_{i} \mathbf{P}+\mathbf{q}_{i \perp}, \quad \mathbf{p}_{i}^{\prime}=x_{i} \mathbf{P}^{\prime}+\mathbf{q}_{i \perp}^{\prime}, \quad \sum_{i} x_{i}=1,(2) \\
& \mathbf{P} \mathbf{q}_{i \perp}=\mathbf{P}^{\prime} \mathbf{q}_{i \perp}^{\prime}=0, \quad \sum_{i} \mathbf{q}_{i \perp}=\sum_{i} \mathbf{q}_{i \perp}^{\prime}=0,
\end{aligned}
$$

where $\mathbf{q}_{i \perp}^{\prime}=\mathbf{q}_{i \perp}-y_{i} \mathbf{Q}_{\perp}$ and $y_{a}=x_{a}-1, y_{b}=x_{b}$, $y_{c}=x_{c}$. The phase space volume corresponding to this parametrization is:

$$
d \Gamma=(2 \pi)^{-6} \frac{d \mathbf{q}_{b \perp} d \mathbf{q}_{c \perp} d x_{b} d x_{c}}{4 x_{a} x_{b} x_{c}} .
$$

According to results of Ref. [7], obtained through relativistic-covariant transformation, the wave function $\Psi$ is related to the wave function in the c.m.s. of the system of three quarks through Melosh matrices [16]:

$$
\Psi=U^{+}\left(p_{a}\right) U^{+}\left(p_{b}\right) U^{+}\left(p_{c}\right) \Psi_{f s s} \Phi\left(\mathbf{q}_{a}, \mathbf{q}_{b}, \mathbf{q}_{c}\right) .
$$

Here we have separated the flavor-spin-space $\left(\Psi_{f s s}\right)$ and spatial $(\Phi)$ parts of the wave function of the quarks in their c.m.s. The Melosh matrices are defined by

$$
U\left(p_{i}\right)=\frac{m_{q}+M_{0} x_{i}+i \epsilon_{l m} \sigma_{l} q_{i m}}{\sqrt{\left(m_{q}+M_{0} x_{i}\right)^{2}+\mathbf{q}_{i \perp}^{2}}},
$$

where $m_{q}$ is the quark mass and $M_{0}$ is invariant mass of the system of initial quarks:

$$
M_{0}^{2}=\left(\sum_{i} p_{i}\right)^{2}=\sum_{i} \frac{\mathbf{q}_{i \perp}^{2}+m_{q}^{2}}{x_{i}} .
$$

In the 3-quark c.m.s.:

$$
M_{0}^{2}=\left(\sum_{i} \omega_{i}\right)^{2}, \quad \omega_{i}=\sqrt{m_{q}^{2}+\mathbf{q}_{i}^{2}}, \quad q_{i z}+\omega_{i}=M_{0} x_{i} .
$$

We construct the flavor-spin-space parts of the wave functions by utilizing the rules [11, 17] that correspond to the classification of the nucleon and nucleon resonances within the group $S U(6) \times O(3)$.

For the final state quarks, the quantities defined in Eqs. (5,8) are expressed through $p_{i}^{\prime}, \mathbf{q}_{i}^{\prime}$, and $M_{0}^{\prime}$.

To study sensitivity to the form of the quark wave function, we employ two forms of the spatial wave function:

$$
\begin{aligned}
& \Phi_{1} \sim \exp \left(-M_{0}^{2} / 6 \alpha_{1}^{2}\right), \\
& \Phi_{2} \sim \exp \left[-\left(\mathbf{q}_{1}^{2}+\mathbf{q}_{2}^{2}+\mathbf{q}_{3}^{2}\right) / 2 \alpha_{2}^{2}\right],
\end{aligned}
$$

that were used, respectively, in Refs. [4, 7, , 8] and [1].

\section{NUCLEON}

The nucleon electromagnetic form factors were described by combining the $3 q$ and $\pi N$ contributions to the nucleon wave function. With the pion loops evaluated according to Ref. [12], the nucleon wave function has the form:

$$
|N>=0.95| 3 q>+0.313 \mid \pi N>,
$$

where the portions of different contributions were found from the condition the charge of the proton be unity: $F_{1 p}(0)=1$.

The values of the quark mass $m_{q}$ and of the parameters $\alpha_{1,2}$ for the wave functions (910) were found from the description of $\mu_{p}=G_{M p}(0)$ and $\mu_{n}=G_{M n}(0)$. Best results,

$$
\mu_{p}=2.86 \frac{e}{2 m_{N}}, \quad \mu_{n}=-1.86 \frac{e}{2 m_{N}},
$$

were obtained with $m_{q}(0)=0.22 \mathrm{GeV}$ and

$$
\alpha_{1}=0.37 \mathrm{GeV}, \quad \alpha_{2}=0.41 \mathrm{GeV} .
$$

The quark mass $m_{q}(0)=0.22 \mathrm{GeV}$ coincides with the value obtained from the description of the spectrum of baryons and mesons and their excited states in the relativized quark model [18, 19].

The parameters (13), that correspond to different forms of wave functions (9) and (10), give very close magnitudes for the mean values of invariant masses and momenta of quarks at $Q^{2}=0:\left\langle M_{0}^{2}>\approx 1.35 \mathrm{GeV}^{2}\right.$ and $<\mathbf{q}_{i}^{2}>\approx 0.1 \mathrm{GeV}^{2}, i=a$, b.c.

A constant value of the quark mass gives rise to rapidly decreasing form factors; for $G_{M p}\left(Q^{2}\right)$ and $G_{M n}\left(Q^{2}\right)$ this is demonstrated in Fig. 1. The wave functions (910) increase as $m_{q}$ decreases. Therefore, to describe the experimental data we have assumed the $Q^{2}$-dependent constituent quark mass. We have used two different parameterizations of this mass:

$$
\begin{aligned}
& m_{q}^{(1)}\left(Q^{2}\right)=\frac{0.22 \mathrm{GeV}}{1+Q^{2} / 56 \mathrm{GeV}^{2}} \\
& m_{q}^{(2)}\left(Q^{2}\right)=\frac{0.22 \mathrm{GeV}}{1+Q^{2} / 18 \mathrm{GeV}^{2}}
\end{aligned}
$$

for the wave functions $\Phi_{1}$ and $\Phi_{2}$, respectively. This resulted in a good description of the nucleon electromagnetic form factors for $Q^{2} \leq 16 \mathrm{GeV}^{2}$.

In Fig. 2 we show separately the pion-cloud contributions. Clearly, at $Q^{2}>2 \mathrm{GeV}^{2}$, all form factors are dominated by the $3 q$-core contribution.

The $Q^{2}$-dependence of the constituent quark mass (Eqs. 14115) is in qualitative agreement with the QCD lattice calculations and Dyson-Schwinger equations [1315], where the running quark mass is generated dynamically. However, we want to point out that there is no direct connection between the functional forms of 

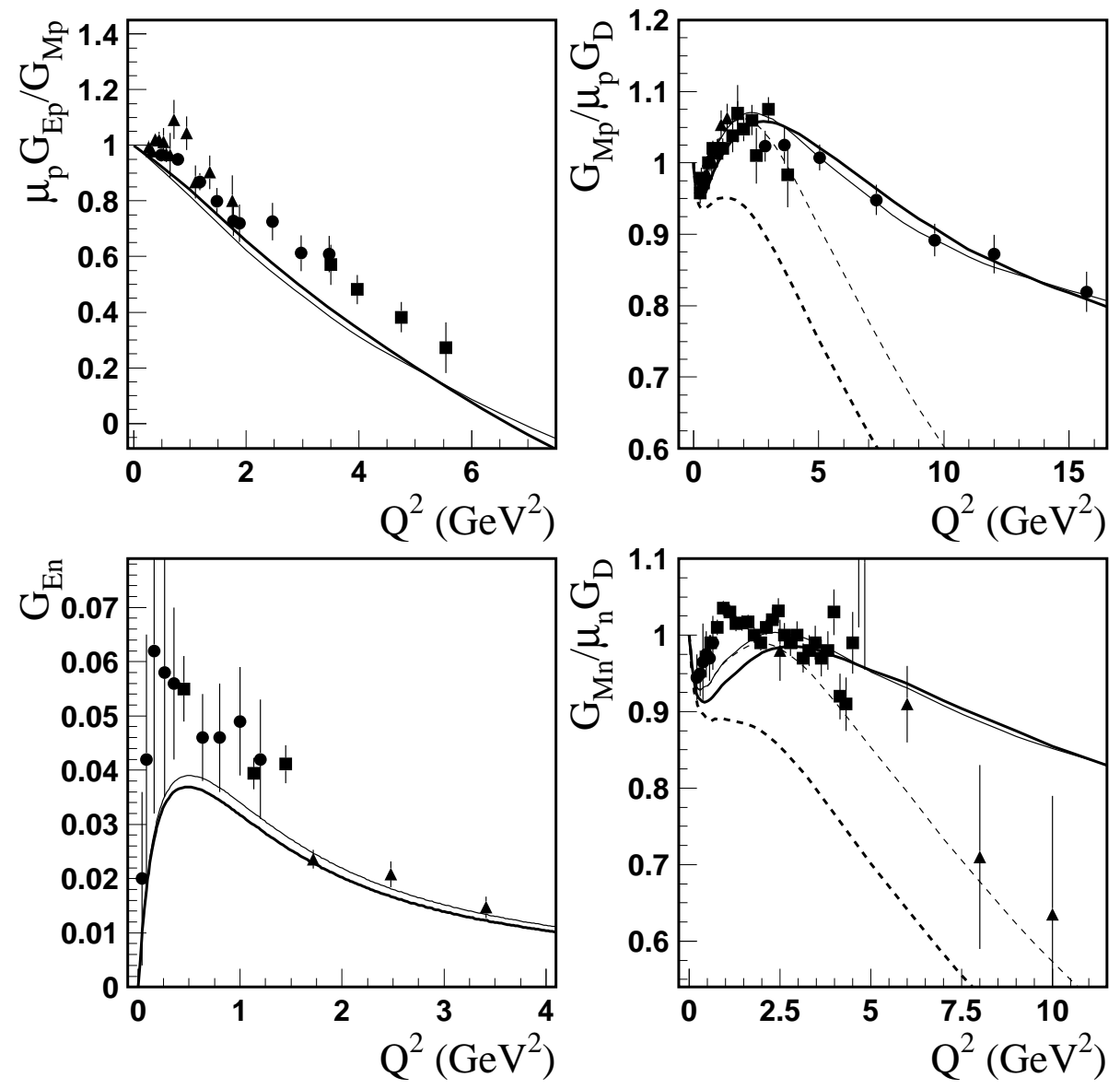

FIG. 1: Nucleon electromagnetic form factors. The curves present the results obtained taking into account two contributions to the nucleon (Eq. 11): the pion-cloud and the $3 q$ core. The thick and thin curves correspond, respectively, to the wave functions (9) and (10). The solid curves are the results obtained with the running quark masses (14) 15) and the dashed curves correspond to the constant quark mass. Data for $G_{E p}\left(Q^{2}\right) / G_{M p}\left(Q^{2}\right)$ are from Refs. 20] - circles, 21] - boxes, 22] - triangles; for $G_{M p}\left(Q^{2}\right)$ : 23] - circles, 24] - boxes, 22] - triangles; for $G_{E n}\left(Q^{2}\right)$ : [25] - circles, [26] - boxes, 27] - triangles; for $G_{M n}\left(Q^{2}\right)$ : [28] - circles, [29] - boxes, 30] - triangles.

these masses. In QCD lattice calculations and DysonSchwinger equations we deal with quarks that do not possess a mass-shell, and the running quark mass is a function of its virtuality, i.e. the quark four-momentum square. In constituent quark models, including the LF approaches [4, 7, 8, 11], the quarks are mass-shell objects (see Eqs. 788). In LF RQM, the virtuality of quarks is characterized by invariant masses of the 3 -quark system: $M_{0}^{2}$ and $M^{\prime 2}{ }_{0}$. Mean values of $M_{0}^{2}$ and $M_{0}^{\prime 2}$ are equal to each other and are increasing with increasing $Q^{2}$.

The mechanism, that generates the running quark mass, can generate also quark anomalous magnetic moments and form factors 31]. In our approach, we have obtained good description of the nucleon electromagnetic form factors without introducing quark anomalous magnetic moments. Introducing quark form factors results in a faster $Q^{2}$ fall-off of form factors and forces $m_{q}\left(Q^{2}\right)$ to drop faster with $Q^{2}$ to describe the data. We found that descriptions, that are very close to those for pointlike quarks and masses (14]15), can be obtained by introduc- ing quark form factors

$$
F_{q}\left(Q^{2}\right)=1 /\left(1+Q^{2} / a_{q}\right)^{2}
$$

with $a_{q}^{(1)}>18 \mathrm{GeV}^{2}$ and $a_{q}^{(2)}>70 \mathrm{GeV}^{2}$ for the wave functions $\Phi_{1}$ and $\Phi_{2}$, respectively. The corresponding quark radii are $r_{q}^{(1)}<r_{N} / 5$ and $r_{q}^{(2)}<r_{N} / 10$, where $r_{N}$ is the mean value of the radii corresponding to $G_{E p}\left(Q^{2}\right)$, $G_{M p}\left(Q^{2}\right)$, and $G_{M n}\left(Q^{2}\right)$. The $Q^{2}$-dependencies of quark masses for minimal values of $a_{q}$ are

$$
\begin{aligned}
& m_{q}^{(1)}\left(Q^{2}\right)=\frac{0.22 \mathrm{GeV}}{1+Q^{2} / 20 \mathrm{GeV}^{2}}, \\
& m_{q}^{(2)}\left(Q^{2}\right)=\frac{0.22 \mathrm{GeV}}{1+Q^{2} / 6 \mathrm{GeV}^{2}} .
\end{aligned}
$$

Therefore, in our approach the quark mass can be in ranges given by Eqs. (14/15) and (17/18). As mentioned above, the results for the nucleon electromagnetic form factors obtained taking into account quark form factors 

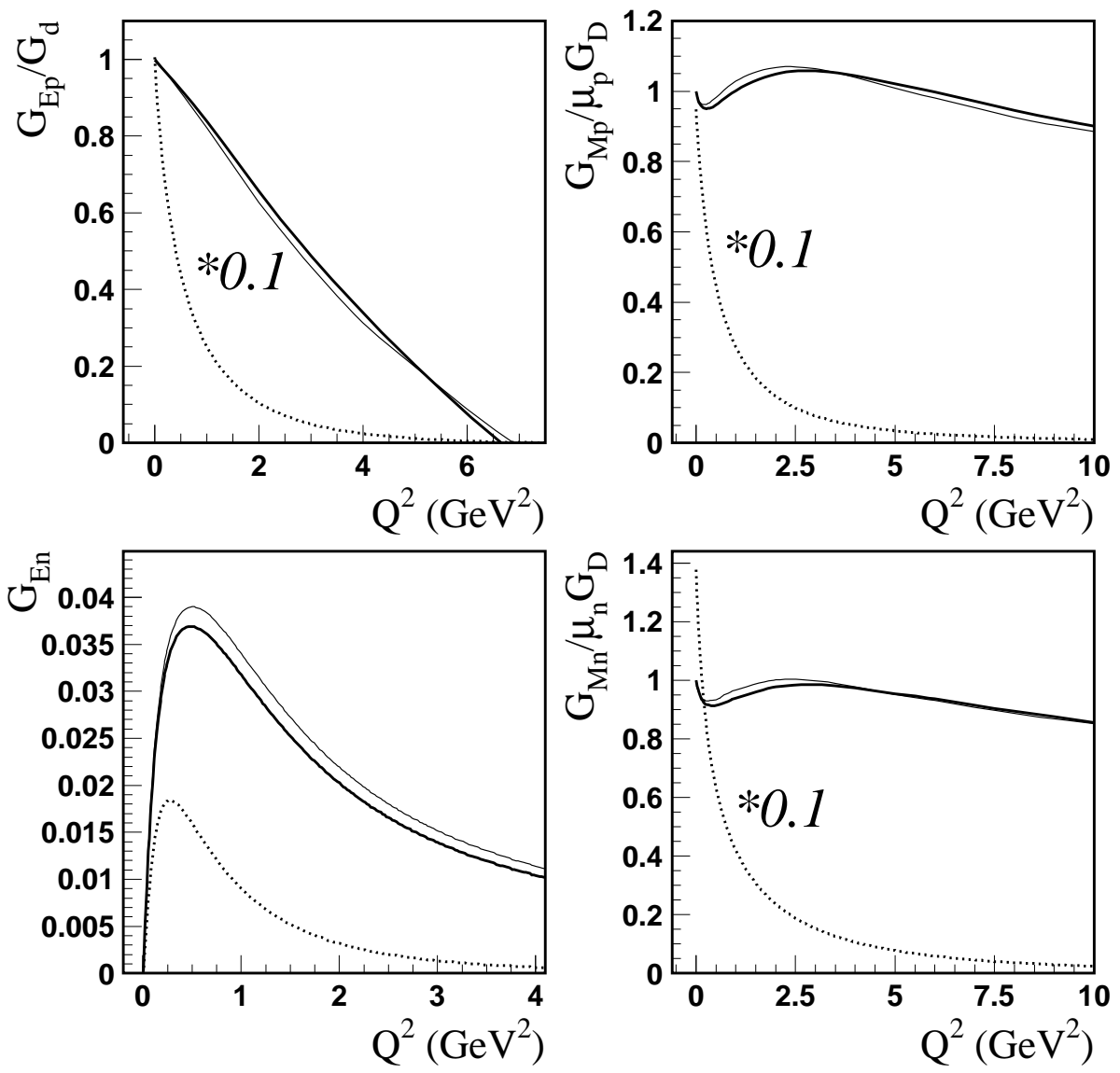

FIG. 2: Nucleon electromagnetic form factors. The legend for the solid curves is as for Fig. 1] The dotted curves are the pioncloud contributions [12]; for all form factors, except $G_{E n}\left(Q^{2}\right)$, the shown results for these contributions should be multiplied by 0.1 .

(16) and masses (17/18) are very close to those for pointlike quarks and masses (14]15). For this reason, they are not shown separately in Figs. 112.

\section{NUCLEON RESONANCES $\boldsymbol{\Delta}(1232) P_{33}$, $\mathbf{N}(1440) P_{11}, \mathbf{N}(1520) D_{13}$, AND $\mathbf{N}(1535) S_{11}$}

No investigations are available that allow for the separation of the $3 q$ and $\pi N$ (or meson-nucleon) contributions to nucleon resonances. Therefore, the weights of the $3 q$ contributions to the resonances:

$$
\left|N^{*}>=c_{N^{*}}\right| 3 q>+\ldots, \quad c_{N^{*}}<1,
$$

are unknown. We estimate these weights by fitting to experimental $\gamma^{*} N \rightarrow N^{*}$ amplitudes. The range of $Q^{2}$ for the fit has been chosen according to available information on the possible meson-cloud contribution to the transition amplitudes. In Ref. [32], the dynamical model has been applied to describe the data on pion electroproduction on proton in the $\Delta(1232) P_{33}$ resonance region at $Q^{2} \leq 4 \mathrm{GeV}^{2}$. As the result, the contribution that can be associated with the meson-cloud con- tribution to $\gamma^{*} N \rightarrow \Delta(1232) P_{33}$ has been found. Unlike for the nucleon, this contribution can not be neglected at $Q^{2}=2-4 \mathrm{GeV}^{2}$ (see Fig. 3). In Ref. 33], the coupled-channel approach has been applied to the description of the pion photoproduction data, and the meson-cloud contribution to the transverse amplitudes for the $N(1440) P_{11}, N(1520) D_{13}$, and $N(1535) S_{11}$ has been found at $Q^{2}=0$. The predicted $Q^{2}$-dependence of this contribution for absolute values of the amplitudes has been presented. According to these results, mesoncloud contributions to $\gamma^{*} N \rightarrow N(1440) \mathrm{P}_{11}, N(1520) D_{13}$, and $N(1535) S_{11}$ are negligible at $Q^{2}>2 \mathrm{GeV}^{2}$. Similar results are obtained for both transverse and longitudinal $\gamma^{*} N \rightarrow N(1440) \mathrm{P}_{11}$ amplitudes via estimation of the $\sigma N$ contribution to this transition [34] (see Fig. 4).

We therefore determine the $3 q$ contribution to the resonances by fitting the experimental amplitudes at $Q^{2}>$ $4 \mathrm{GeV}^{2}$ for the $\Delta(1232) P_{33}$ and at $Q^{2}=2.5-4.5 \mathrm{GeV}^{2}$ for the $N(1440) P_{11}, N(1520) D_{13}$, and $N(1535) S_{11}$, assuming that at these $Q^{2}$ the transition amplitudes are dominated by the $3 q$ contribution. The results are shown in Figs. 3 6, For the $\Delta(1232) P_{33}$ and $N(1440) P_{11}$, we present also the results where the $3 q$-core is complemented, respectively, by the meson-cloud [32] and $\sigma N$ 

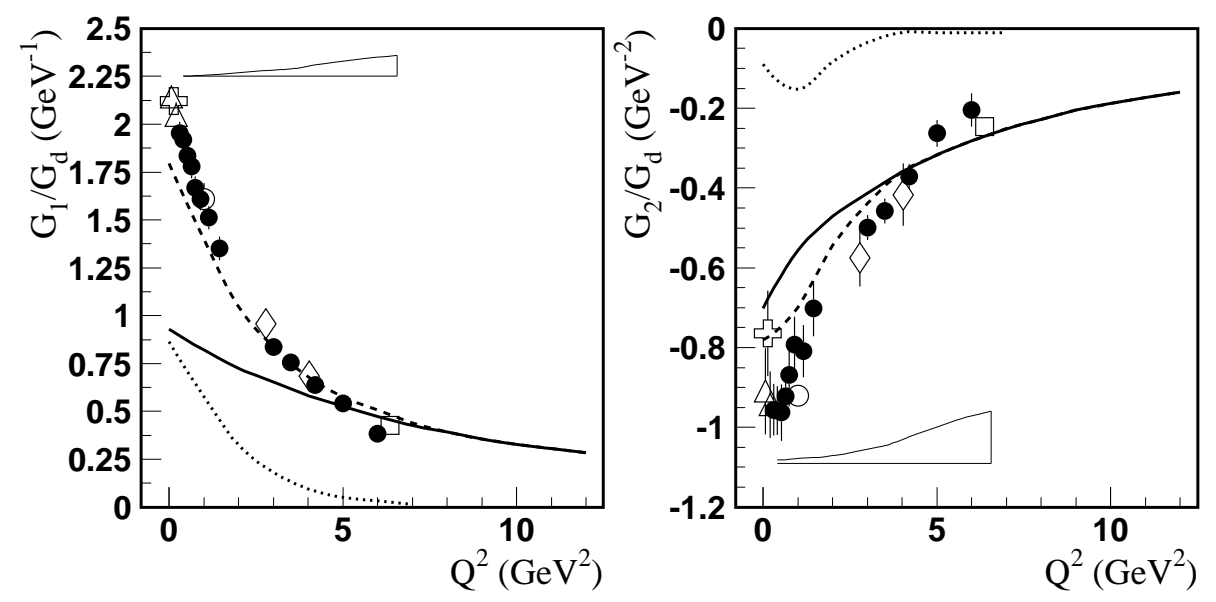

FIG. 3: The $\gamma^{*} p \rightarrow \Delta(1232) \mathrm{P}_{33}$ transition form factors. The solid curves correspond to the LF RQM predictions; the weight factors for the $3 q$ contributions to the $\Delta(1232) \mathrm{P}_{33}$ are $c_{N^{*}}^{(1)} \approx c_{N^{*}}^{(2)}=0.53 \pm 0.04$ for the wave functions of Eqs. (9) and (10). The dotted curves correspond to the meson-cloud contributions obtained in the dynamical model [32]. The dashed curves present the sum of the $3 q$ and meson-cloud contributions. Solid circles are the amplitudes extracted from the CLAS pion electroproduction data 35], bands represent model uncertainties of these results. The results from other experiments are: open triangles [36 38], open crosses [39 41], open rhombuses [42], open boxes [43], and open circles [44, 45].
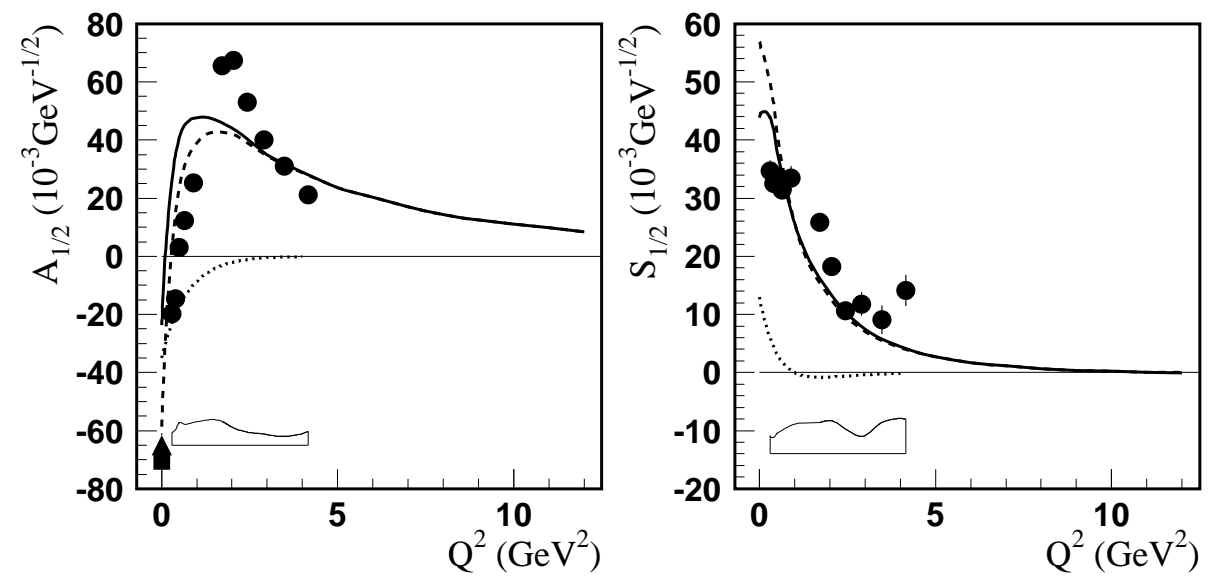

FIG. 4: The $\gamma^{*} p \rightarrow N(1440) \mathrm{P}_{11}$ transition helicity amplitudes. The solid curves are the LF RQM predictions obtained with the weight factors $c_{N^{*}}^{(1)}=0.73 \pm 0.05$ and $c_{N^{*}}^{(2)}=0.77 \pm 0.05$ for the $3 q$ contribution to the $N(1440) \mathrm{P}_{11}$. The dotted curves correspond to the $\sigma N$ contribution [34]. The dashed curves present the sum of the $3 q$ and $\sigma N$ contributions. Solid circles are the amplitudes extracted from the CLAS pion electroproduction data [35], bands represent model uncertainties of these results. The full box at $Q^{2}=0$ is the amplitude extracted from CLAS $\pi$ photoproduction data [46]. The full triangle at $Q^{2}=0$ is the RPP estimate [47].

34] contributions. These contributions significantly improve the agreement with experimental amplitudes at low $Q^{2}$.

Here we comment on the amplitudes presented in Figs. 3[6. As it is shown in Refs. 9, 54], there are difficulties in the utilization of the LF approaches [4, 7, 8, 11] for hadrons with spins $J \geq 1$. These difficulties are not present if Eq. (10) is used to calculate only those matrix elements that correspond to $S_{z}^{\prime}=J[9]$. This restricts the number of transition form factors that can be investigated for the resonances $\Delta(1232) P_{33}$ and $N(1520) D_{13}$. As can be seen from Eqs. (A15, A16), the matrix ele- ments with $S_{z}^{\prime}=\frac{3}{2}$ relate to only two transition form factors: $G_{1}\left(Q^{2}\right)$ and $G_{2}\left(Q^{2}\right)$. Consequently, we can not present the results in terms of transition helicity amplitudes for the $\Delta(1232) P_{33}$ and $N(1520) D_{13}$, while the resonances with $J=\frac{1}{2}$, i.e. $N(1440) P_{11}$ and $N(1535) S_{11}$, are presented in terms of these amplitudes.

Using eqs. A22 - A27), the transition form factors $G_{1,2}\left(Q^{2}\right)$ for the $\Delta(1232) P_{33}$ and $N(1520) D_{13}$ can be related to the transition helicity amplitudes. For $G_{1}\left(Q^{2}\right)$, the relation has simple form:

$$
G_{1}\left(Q^{2}\right)=\mp \frac{m_{N^{*}}}{2 X Q_{ \pm}}\left(A_{1 / 2} \pm \frac{1}{\sqrt{3}} A_{3 / 2}\right)
$$



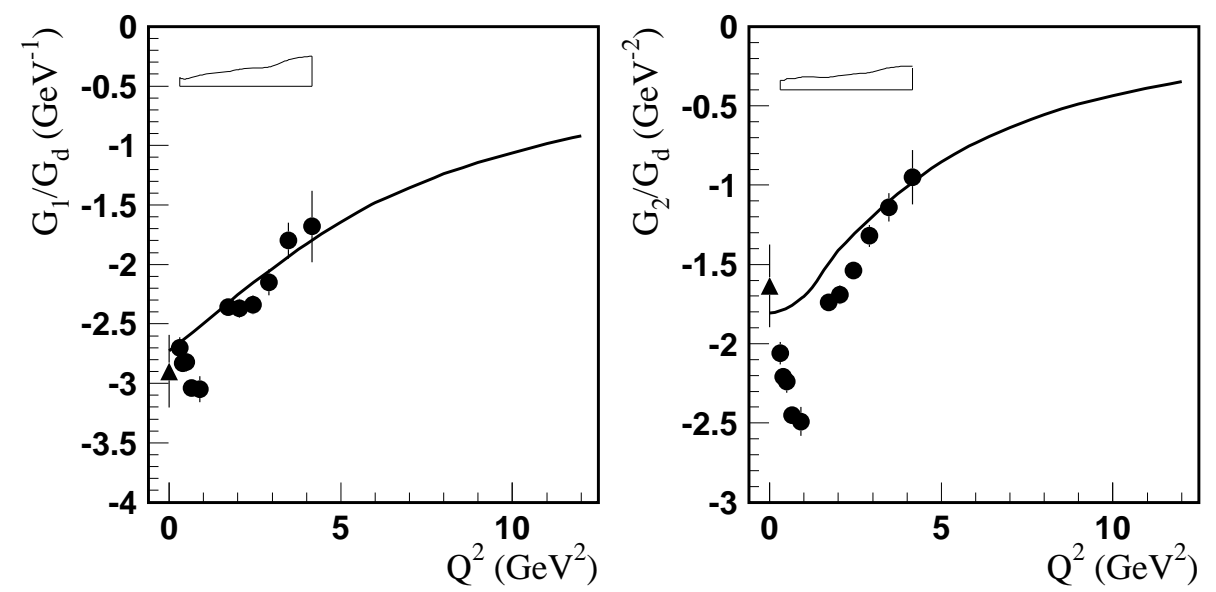

FIG. 5: The $\gamma^{*} p \rightarrow N(1520) \mathrm{D}_{13}$ transition form factors. $c_{N^{*}}^{(1)}=0.78 \pm 0.06, c_{N^{*}}^{(2)}=0.82 \pm 0.06$. Other legend is as for Fig. 4]
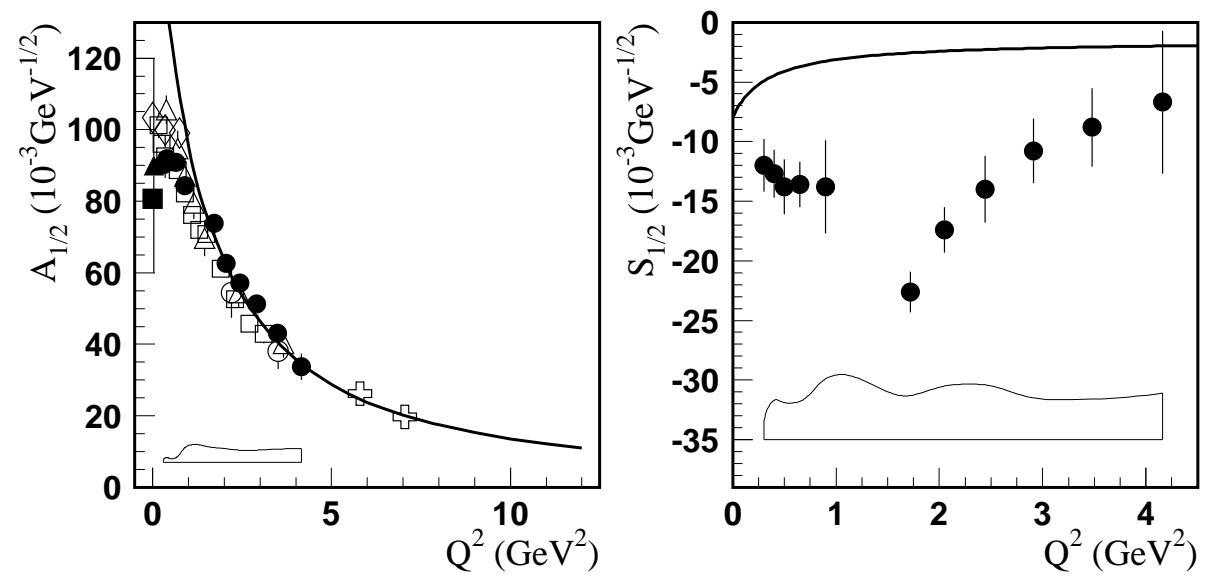

FIG. 6: The $\gamma^{*} p \rightarrow N(1535) \mathrm{S}_{11}$ transition helicity amplitudes. The open triangles [48], open boxes [49], and open rhombuses [52, 53] are the amplitudes extracted from the JLab/Hall B $\eta$ electroproduction data; the open circles [50] and open crosses [51] are the amplitudes extracted from the JLab/Hall C $\eta$ electroproduction data. $c_{N^{*}}^{(1)}=0.88 \pm 0.03, c_{N^{*}}^{(2)}=0.94 \pm 0.03$. Other legend is as for Fig. 4

where $Q_{ \pm}$and $X$ are defined by eqs. A14, A24 and the upper and lower symbols correspond, respectively, to the $\Delta(1232) P_{33}$ and $N(1520) D_{13}$. For the $\Delta(1232) P_{33}$, it is useful to also present the following relation:

$$
G_{1}\left(Q^{2}\right)=\sqrt{\frac{3}{2}} \frac{m_{N^{*}}\left(m_{N^{*}}+m_{N}\right)}{m_{N} Q_{+}}\left(G_{M}\left(Q^{2}\right)-G_{E}\left(Q^{2}\right)\right),
$$

where $G_{M}\left(Q^{2}\right)$ and $G_{E}\left(Q^{2}\right)$ are the form factors defined in Ref. [55].

We note that the predictions obtained with different wave functions (910) and corresponding masses (14/15), as well as the predictions found taking into account quark form factors (16) and masses (17/18) only differ in the weight factors $c_{N^{*}}$ for the wave functions (9) and (10); these factors are given in the figure captions. The LF RQM predictions for resonances are therefore presented by a single (thick solid) curve.

We also note that the nucleon and $\Delta(1232) P_{33}$ as well as the $N(1440) P_{11}$ are considered as members of the $\left[56,0^{+}\right]$and $\left[56,0^{+}\right]_{R}$ multiplets, respectively. The $N(1520) D_{13}$ is taken as the state ${ }^{2} 8_{3 / 2}$ of the multiplet $\left[70,1^{-}\right]$and the $N(1535) S_{11}$ as a mixture of the states ${ }^{2} 8_{1 / 2}$ and ${ }^{4} 8_{1 / 2}$ in this mutiplet:

$$
N(1535) S_{11}=\left.\cos \theta_{\mathrm{S}}\right|^{2} 8_{1 / 2}>-\left.\sin \theta_{\mathrm{S}}\right|^{4} 8_{1 / 2}>.
$$

Here we use the notation ${ }^{2 S+1} S U(3)_{J}$, which gives the assignment according to the $S U(3)$ group, $J$ is the spin of the resonance, and $S$ is the total spin of quarks. The mixing angle is taken equal to $\theta_{S}=-31^{\circ}$ as found from the hadronic decays [56, 57]. The transition $\gamma^{*} p \rightarrow{ }^{4} 8_{1 / 2}$, which is forbidden in the single quark transition model [58], turned out very small compared to $\gamma^{*} p \rightarrow{ }^{2} 8_{1 / 2}$ in the LF RQM too. Therefore, the $\gamma^{*} p \rightarrow N(1535) S_{11}$ amplitudes are determined mainly by the first term in Eq. (22). 


\section{SUMMARY}

We have described the nucleon electromagnetic form factors in a wide range of $Q^{2}$ by complementing the $3 q$ core contribution with contribution of the pion cloud, and assuming the constituent quark mass to decrease with increasing $Q^{2}$. The pion-cloud contribution is negligible at $Q^{2}>2 \mathrm{GeV}^{2}$, but it is important to describe the neutron electric form factor and the dip in the magnetic form factors at very small $Q^{2}$. The decreasing quark mass allowed us to compensate the rapidly falling form factors with increasing $Q^{2}$. The $Q^{2}$-dependent quark mass is in qualitative agreement with results from QCD lattice and Dyson-Schwinger equations. The mechanism, that generates the running quark mass within these approaches, can also produce quark form factors which result in a faster fall-off of the nucleon form factors. This, in turn, forces $m_{q}\left(Q^{2}\right)$ to drop faster with $Q^{2}$ in order to describe the data. From the description of the nucleon electromagnetic form factors, we have found empirically the boundaries for the quark form factors and the corresponding boundaries for $m_{q}\left(Q^{2}\right)$.

With the LF RQM specified via description of the nucleon electromagnetic form factors, we have predicted the quark core contribution to the electroexcitation amplitudes of the resonances $\Delta(1232) P_{33}, N(1440) P_{11}$, $N(1520) D_{13}$, and $N(1535) S_{11}$ up to $Q^{2}=12 \mathrm{GeV}^{2}$, where the weight factor of the $3 q$ contribution to the resonance occurs as the only parameter. This parameter was found by fitting to experimental amplitudes in a $Q^{2}$ range, where the meson-cloud contribution is expected to be negligible. The important feature of our predictions is the fact that at these $Q^{2}$ we describe both amplitudes investigated for each resonance by fitting a single parameter.

For the $\Delta(1232) P_{33}$ and $N(1440) P_{11}$, we also present the results where the $3 q$-core is complemented, respectively, by the meson-cloud contribution found in the dynamical model 32] and the $\sigma N$ contribution found in Ref. [34] . These contributions significantly improve the agreement with experimental amplitudes at low $Q^{2}$.

\section{ACKNOWLEDGMENTS}

We acknowledge valuable communications with T.S.H. Lee and C.D. Roberts. This work was supported by the US Department of Energy under contract DEAC05-06OR23177 and the Department of Education and Science of Republic of Armenia, Grant-11-1C015.

\section{APPENDIX. THE RELATIONS BETWEEN THE MATRIX ELEMENTS (1) AND THE $\gamma^{*} N \rightarrow N\left(N^{*}\right)$ FORM FACTORS AND TRANSITION HELICITY AMPLITUDES}

For the nucleon, the matrix elements (11) are related to the form factors in the following way:

$$
\begin{gathered}
\frac{1}{2 P_{z}}<N, \frac{1}{2}\left|J_{e m}^{0,3}\right| N, \frac{1}{2}>\left.\right|_{P_{z} \rightarrow \infty}=F_{1}, \\
\frac{1}{2 P_{z}}<N, \frac{1}{2}\left|J_{e m}^{0,3}\right| N,-\frac{1}{2}>\left.\right|_{P_{z} \rightarrow \infty}=-\frac{Q}{2 m_{N}} F_{2},
\end{gathered}
$$

where $F_{1}\left(Q^{2}\right)$ and $F_{2}\left(Q^{2}\right)$ are the Dirac and Pauli form factors: $F_{1 p}(0)=1, F_{2 N}(0)=\kappa_{N}$, the nucleon anomalous magnetic moment. The Sachs form factors are:

$$
G_{M}\left(Q^{2}\right)=F_{1}+F_{2}, \quad G_{E}\left(Q^{2}\right)=F_{1}-\frac{Q^{2}}{4 m_{N}^{2}} F_{2}
$$

For the resonances with $J^{P}=\frac{1}{2}^{ \pm}$:

$$
\begin{gathered}
\frac{1}{2 P_{z}}<N^{*}, \frac{1}{2}\left|J_{\text {em }}^{0,3}\right| N, \frac{1}{2}>\left.\right|_{P_{z} \rightarrow \infty}=Q^{2} G_{1}, \\
\frac{1}{2 P_{z}}<N^{*}, \frac{1}{2}\left|J_{\text {em }}^{0,3}\right| N,-\frac{1}{2}>\left.\right|_{P_{z} \rightarrow \infty} \\
=\frac{ \pm m_{N^{*}}-m_{N}}{2} Q G_{2},
\end{gathered}
$$

where the upper and lower symbols correspond, respectively, to $J^{P}=\frac{1}{2}^{+}$and $\frac{1}{2}^{-}$resonances, and the form factors are defined by [1, [59]:

$$
\begin{gathered}
<N^{*}\left|J_{e m}^{\mu}\right| N>\equiv e \bar{u}\left(P^{\prime}\right)\left(\begin{array}{c}
1 \\
\gamma_{5}
\end{array}\right) \tilde{J}^{\mu} u(P), \\
\tilde{J}^{\mu}=\left(\not k k^{\mu}-k^{2} \gamma^{\mu}\right) G_{1}+\left[\not k \mathcal{P}^{\mu}-(\mathcal{P} k) \gamma^{\mu}\right] G_{2},
\end{gathered}
$$

$\mathcal{P} \equiv \frac{1}{2}\left(P^{\prime}+P\right), u(P), u\left(P^{\prime}\right)$ are the Dirac spinors. The relations between the $\gamma^{*} N \rightarrow N^{*}$ helicity amplitudes and the form factors $G_{1}\left(Q^{2}\right), G_{2}\left(Q^{2}\right)$ are following:

$$
\begin{aligned}
& A_{\frac{1}{2}}=b\left[2 Q^{2} G_{1}-\left(m_{N^{*}}^{2}-m_{N}^{2}\right) G_{2}\right] \\
& S_{\frac{1}{2}}= \pm b \frac{|\mathbf{k}|}{\sqrt{2}} \tilde{S}_{\frac{1}{2}} \\
& \tilde{S}_{\frac{1}{2}}=2\left(m_{N^{*}} \pm m_{N}\right) G_{1}+\left(m_{N^{*}} \mp m_{N}\right) G_{2} \\
& b \equiv e \sqrt{\frac{Q_{\mp}}{8 m_{N}\left(m_{N^{*}}^{2}-m_{N}^{2}\right)}} \\
& |\mathbf{k}|=\frac{\sqrt{Q_{+} Q_{-}}}{2 m_{N^{*}}} \\
& Q_{ \pm} \equiv\left(m_{N^{*}} \pm m_{N}\right)^{2}+Q^{2}
\end{aligned}
$$

For the resonances with $J^{P}=\frac{3}{2}^{ \pm}$: 


$$
\begin{gathered}
\frac{1}{2 P_{z}}<N^{*}, \frac{3}{2}\left|J_{e m}^{0,3}\right| N, \frac{1}{2}>\left.\right|_{P_{z} \rightarrow \infty}= \\
-\frac{Q}{\sqrt{2}}\left[G_{1}\left(Q^{2}\right)+\frac{ \pm m_{N^{*}}-m_{N}}{2} G_{2}\left(Q^{2}\right)\right], \\
\frac{1}{2 P_{z}}<N^{*}, \frac{3}{2}\left|J_{e m}^{0,3}\right| N,-\frac{1}{2}>\left.\right|_{P_{z} \rightarrow \infty}= \\
\frac{Q^{2}}{2 \sqrt{2}} G_{2}\left(Q^{2}\right),
\end{gathered}
$$

and the form factors are defined by [1, [59]:

$$
\begin{gathered}
<N^{*}\left|J_{e m}^{\mu}\right| N>\equiv e \bar{u}_{\nu}\left(P^{\prime}\right)\left(\begin{array}{c}
\gamma_{5} \\
1
\end{array}\right) \Gamma^{\nu \mu} u(P) \\
\Gamma^{\nu \mu}\left(Q^{2}\right)=G_{1} \mathcal{H}_{1}^{\nu \mu}+G_{2} \mathcal{H}_{2}^{\nu \mu}+G_{3} \mathcal{H}_{3}^{\nu \mu} \\
\mathcal{H}_{1}^{\nu \mu}=\not k g^{\nu \mu}-k^{\nu} \gamma^{\mu} \\
\mathcal{H}_{2}^{\nu \mu}=k^{\nu} P^{\prime \mu}-\left(k P^{\prime}\right) g^{\nu \mu} \\
\mathcal{H}_{3}^{\nu \mu}=k^{\nu} k^{\mu}-k^{2} g^{\nu \mu}
\end{gathered}
$$

where $u_{\nu}\left(P^{\prime}\right)$ is the generalized Rarita-Schwinger spinor. The relations between the $\gamma^{*} N \rightarrow N^{*}$ helicity ampli- tudes and form factors for the $J^{P}=\frac{3}{2}^{ \pm}$resonances are following:

$$
\begin{aligned}
& A_{1 / 2}=h_{3} X, \quad A_{3 / 2}= \pm \sqrt{3} h_{2} X, \\
& S_{1 / 2}=h_{1} \frac{|\mathbf{k}|}{\sqrt{2} m_{N^{*}}} X, \\
& X \equiv e \sqrt{\frac{Q_{\mp}}{48 m_{N}\left(m_{N^{*}}^{2}-m_{N}^{2}\right)}},
\end{aligned}
$$

where

$$
\begin{aligned}
& h_{1}\left(Q^{2}\right)= \pm 4 m_{N^{*}} G_{1}\left(Q^{2}\right)+4 m_{N^{*}}^{2} G_{2}\left(Q^{2}\right)+ \\
& \quad 2\left(m_{N^{*}}^{2}-m_{N}^{2}-Q^{2}\right) G_{3}\left(Q^{2}\right), \\
& h_{2}\left(Q^{2}\right)=-2\left( \pm m_{N^{*}}+m_{N}\right) G_{1}\left(Q^{2}\right)- \\
& \left(m_{N^{*}}^{2}-m_{N}^{2}-Q^{2}\right) G_{2}\left(Q^{2}\right)+2 Q^{2} G_{3}\left(Q^{2}\right), \\
& h_{3}\left(Q^{2}\right)=\mp \frac{2}{m_{N^{*}}}\left[Q^{2}+m_{N}\left( \pm m_{N^{*}}+m_{N}\right)\right] G_{1}\left(Q^{2}\right)+ \\
& \left(m_{N^{*}}^{2}-m_{N}^{2}-Q^{2}\right) G_{2}\left(Q^{2}\right)-2 Q^{2} G_{3}\left(Q^{2}\right) .
\end{aligned}
$$

[1] I. G. Aznauryan, V. D. Burkert, Prog. Part. Nucl. Phys. 67, 1 (2012), arXiv:1109.1720, 2011.

[2] L. Tiator, D. Drechsel, S.S. Kamalov, and M. Vanderhaeghen, arXiv:1109,6745, 2011.

[3] S. D. Drell and T. M. Yan, Phys. Rev. Lett. 24, 181 (1970).

[4] V. B. Berestetskii and M. V. Terent'ev, Sov. J. Nucl. Phys., 24, 1044 (1976); 25, 347 (1977).

[5] S. J. Brodsky and S. D. Drell, Phys. Rev. D 22, 2236 (1980).

[6] L. A. Kondratyuk and M. V. Terent'ev, Yad. Fiz., 31, 1087 (1980).

[7] I. G. Aznauryan, A. S. Bagdasaryan, and N. L. TerIsaakyan, Phys. Lett. B 112, 393 (1982); Yad. Fiz. 36, 1278 (1982).

[8] I. G. Aznauryan, Phys. Lett. B 316, 391 (1993); Z. für Phys. A346, 297 (1993).

[9] I. G. Aznauryan and A. S. Bagdasaryan, Sov. J. Nucl. Phys. 41, 158 (1985).

[10] I. G. Aznauryan, Phys. Rev. C 76, 025212 (2007).

[11] S. Capstick and B. D. Keister, Phys. Rev. D 51, 3598 (1995).

[12] G. A. Miller, Phys. Rev. C 66, 032201 (2002).

[13] P. O. Bowman et al., Phys. Rev. D 71054507 (2005).

[14] M.S. Bhagwat et al., Phys. Rev. C 68015203 (2003).

[15] M.S. Bhagwat and P. C. Tandy, AIP. Conf. Proc. 842 225 (2006).

[16] H. J. Melosh, Phys. Rev. D 9, 1095 (1974).

[17] R. Koniuk and N. Isgur, Phys. Rev. D 21, 1868 (1980).

[18] S. Godfrey and N. Isgur, Phys. Rev. D 32, 189 (1985).

[19] S. Capstick and N. Isgur, Phys. Rev. D 34, 2809 (1986).

[20] M. K. Jones et al., Phys. Rev. Lett. 84, 1398 (2000).

[21] O. Gayou et al., Phys. Rev. Lett. 88, 092301 (2002).

[22] L. E. Price et al., Phys. Rev. D 4, 45 (1971).
[23] A. F. Sill et al., Phys. Rev. D 48, 29 (1993).

[24] W. Bartel et al., Nucl. Phys. B 58, 429 (1973).

[25] R. Schiavilla and I. Sick, Phys. Rev. C 64, 041002 (2001).

[26] R. Madey et al., Phys. Rev. Lett. 91, 122002 (2003).

[27] S. Riordan et al., Phys. Rev. Lett. 105, 262302 (2010).

[28] B. Anderson et al., Phys. Rev. C 75, 043003 (2007).

[29] J. Lachniet et al., CLAS collaboration, Phys. Rev. Lett. 102, 192001 (2009).

[30] S. Rock et al., Phys. Rev. Lett. 49, 1139 (1982).

[31] L. Chang, Y.-X. Liu, and C. D. Roberts, Phys. Rev. Lett. 106072001 (2011).

[32] T. Sato and T.-S. H. Lee, Phys. Rev. C 63055201 (2001).

[33] B. Juliá-Díaz, T.-S. H. Lee, A. Matsuyama, and T. Sato, Phys. Rev. C 77045205 (2008).

[34] I. T. Obukhovsky, A. Faessler, D. K. Fedorov et al., Phys. Rev. D 84014004 (20011).

[35] I. G. Aznauryan, et al., CLAS collaboration, Phys. Rev. C80, 055203 (2009); Phys.Rev. C 78, 045209 (2008)

[36] S. Stave et al., Eur. Phys. J. A 30471 (2006).

[37] N.F. Sparveris et al., Phys. Lett. B 651102 (2007).

[38] S. Stave et al., Phys. Rev. C 78025209 (2008).

[39] C. Mertz et al., Phys. Rev. Lett. 862963 (2001).

[40] C. Kunz et al., Phys. Lett. B 56421 (2003).

[41] N.F. Sparveris et al., Phys. Rev. Lett. 94022003 (2005).

[42] V.V. Frolov et al., Phys. Rev. Lett. 8245 (1999).

[43] A.N. Villano et al., Phys. Rev. C 80035203 (2009).

[44] J. J. Kelly et al., Phys. Rev. Lett. 95102001 (2005).

[45] J. J. Kelly et al., Phys. Rev. C 75025201 (2007).

[46] M. Dugger et al., CLAS Collaboration, Phys. Rev. C 79 065206 (2009).

[47] K. Nakamura et al. [Particle Data Group], Journal of Phys. G 371 (2010).

[48] R. Thompson et al., CLAS Collaboration, Phys. Rev. Lett. 86, 1702 (2001). 
[49] H. Denizli et al., CLAS Collaboration, Phys. Rev. C 76 015204 (2007).

[50] C.S. Armstrong et al., Phys. Rev. D 60052004 (2009).

[51] M.M. Dalton et al., Phys. Rev. C 80015205 (2009).

[52] I.G. Aznauryan et al., Phys. Rev. C71 015201 (2005).

[53] I.G. Aznauryan, Phys. Rev. C68 065204 (2003).

[54] B. D. Keister, Phys. Rev. D49, 1500 (1994).

[55] H. F. Jones and M. D. Scadron, Ann. Phys. 81 (1973) 1.
[56] N. Isgur and G. Karl, Phys. Lett. B72 109 (1977).

[57] A.J.G. Hey, P.J. Litchfield, and R.J. Cashmore, Nucl. Phys. B95 516 (1975).

[58] V.D. Burkert et al., Phys. Rev. C 67035204 (2003).

[59] R.C.E. Devenish, T.S. Eisenschitz, and J.G. Körner, Phys. Rev. D 143063 (1976). 